\title{
Attitudinal Effects of Character-Based Versus Competence-Based Negative Political Communications
}

\author{
Pamela M. Homer \\ California State University, Long Beach \\ Rajeev Batra \\ University of Michigan
}

\begin{abstract}
Many political communications are negative in nature, but not much is known of the situations in which such communications are more damaging to the targeted political candidate. We hypothesized and experimentally demonstrated that (a) negative political communications are more successful in damaging overall voter attitudes toward the targeted candidate than positive communications are in raising such attitudes, (b) such negative communications are more effective in changing those beliefs about the targeted candidate that concern character (likability and trustworthiness) than in modifying competence (expertise-related) beliefs, and (c) these attitude-changing effects are greater when voters form their overall attitudes toward the targeted candidate more on the basis of character beliefs than competence ones. Implications of these results are discussed for public policy and for attitude theory.
\end{abstract}

Because political advertising is undoubtedly a significant element in the total political process (Jamieson, 1984), a better understanding of the mechanisms by which consumers process political advertising is clearly worth seeking in part because it may add to our knowledge about advertising in general. One very visible characteristic of political advertising is that it is increasingly negative in nature: It has been estimated that between $30 \%$ and $50 \%$ of all advertising in past political campaigns was negative (Taylor, 1986). Such negative advertisements deal with an opponent's frailties rather than the candidate's own views toward public issues. In other words, although a candidate using a

Requests for reprints should be sent to Pamela M. Homer, College of Business Administration, California State University, Long Beach, 1250 Bellflower Boulevard, Long Beach, CA 90840-8501. 
positive ad tries to communicate "what's good about me," a candidate using a negative ad communicates "what's bad about my opponent" (JohnsonCartee \& Copeland, 1991, p. 29). Moreover, negative advertising seeks to move message recipients away from the targeted candidate and may mention nothing at all about the sponsoring candidate's attributes. It focuses predominantly on degrading perceptions of the opponent to the advantage of the source. James and Hensel's (1991) review noted that negative advertising is characterized most clearly as that in which consumers perceive a violation of "fair play" standards and perceive derogatory, image damaging, malicious, or vicious references about the attacked competitor (p. 56).

Considering the extent to which these negative political ads are employed, one would assume that they are always effective at persuading voters. However, the evidence concerning negative political advertising is actually quite equivocal, and it is often based only on one-shot case studies. A few researchers have indeed examined negative advertisements more systematically, but even in those studies we find different opinions concerning their effectiveness. Although some researchers suggest that negative political ads are informative and possess significant educational potential (Surlin \& Gordon, 1977), Merritt (1984) suggested that the negative information contained in negative political advertisements lacks credibility, reducing their effectiveness. Evidence suggests that such tactics can often produce a backlash effect on the image of the attacker (e.g., Garramone, 1984; Merritt, 1984), thus limiting their counterattitudinal effectiveness. Tinkham and Weaver-Lariscy (1991) found that, of 272 Congressional races in 1982, only about $24 \%$ of the candidates (both incumbents and challengers) using negative appeals won, whereas $76 \%$ of those using positive appeals did so. Some authors have also concluded that the general public finds negative advertising offensive and less believable (see reviews in James \& Hensel, 1991, and Johnson-Cartee \& Copeland, 1991).

Given these differing opinions on the attitude-changing effectiveness of such negative political advertising, it is useful to develop a theoretical (contingency) framework that could help us better understand situational variations in the effectiveness of such negative communications: Are there some important moderator variables that limit the effectiveness of negative information in changing attitudes?

\section{LITERATURE REVIEW AND HYPOTHESES}

\section{Positive Versus Negative Information}

Because we are discussing political communications that contain negative information, previous research on the impact of positive versus negative information on evaluations is clearly relevant. It has been shown that ratings of 
people described by positive and negative adjectives are more negative than would be expected by averaging the individual scale values (e.g., Anderson, 1965). Similarly, the modifying capacity of negative adjectives appears to be greater than that of positive adjectives (Feldman, 1966), and Birnbaum (1972) demonstrated that evaluations of pairs of immoral acts most resembled the worst of the two acts. Richey, Koenigs, Richey, and Fortin (1975) found that a single report of an individual's negative behavior outweighed reports of five positive behaviors. Some studies in the consumer research area also support the notion that negative information has a greater influence on attitudes and purchase intentions toward products than otherwise equivalent positive information (Mizerski, 1982; Weinburger, Allen, \& Dillon, 1981). For a comprehensive review of the explanations of negativity biases in impression formation, see Skowronski and Carlston (1989).

Research on this "negativity effect" also exists in the political studies field. Utilizing data from the Center for Political Studies' National Election Studies between 1968 and 1980, Lau (1985) claimed to find a negativity effect in political behavior similar to that just described. Earlier, Lau (1982) found that evaluations of presidential candidates in 1968, 1972, and 1980 were more strongly shaped by negative information than by positive information. That is, these evaluations fell more sharply with negative information than they rose with positive information-the effects were not symmetric. However, because Lau's studies used correlational, not experimental, designs, the negativity effect in the political studies field requires more study using better controlled experimental studies. Such an experimental study was one of our goals. We thus hypothesize that:

H1: Negative information will lead to greater attitude change (in a downward direction) than will positive information (in an upward direction).

The literature review thus far has examined the main effect of negative advertising. We turn now to possible interaction effects.

\section{Dimensionalizing Beliefs About Sources: Competence and Character}

Research has shown that political candidates are evaluated by voters on two separate belief dimensions, which we will henceforth call character and competence. Garramone, Steele, and Pinkleton (1991) reported a two-dimensional factor structure for candidate evaluations: a Competence index containing attributes such as decisiveness and intelligence and a Character index including attributes of friendly, warm, likable, honest, and trustworthy. These indices coincide with the two trait dimensions of Competence (e.g., hardworking and 
qualified) and Integrity (e.g., compassionate and dishonest) reported by Lodge, McGraw, and Stroh (1989). Similar dimensions have emerged in earlier political science literature: Miller, Wattenberg, and Malanchuk (1986) also reported two cognitive trait dimensions of Competence (e.g., hardworking and qualified) and Integrity (e.g., dishonest and compassionate), whereas Marcus (1988) found two factors in emotional self-report data called Performance/ Command and Moral Leadership. Kinder (1986) reported four dimensions including Competence (e.g., hardworking and qualified), Leadership (e.g., strong and inspiring) and two dimensions equivalent to others' concept of integrity: Integrity (e.g., dishonest) and Empathy (e.g., compassionate).

Integrity and empathy are also similar to dimensions of candidate evaluation found in the literature on source characteristics, in which the criteria used by message recipients to evaluate message sources were dimensionalized. Although some past source credibility research found sources to be evaluated on criteria such as expertness and trustworthiness (e.g., Hass, 1981; Hovland, Janis, \& Kelley, 1953), other research (e.g., McGuire, 1969) devoted considerable attention to the concepts of a source's attractiveness, familiarity, likability, and similarity. Integrating these past efforts, Ohanian (1990) dimensionalized such source appraisals (using factor analyses) on scales designed to measure separately a source's perceived expertise, trustworthiness, and attractiveness.

Given these theoretical and empirical distinctions, it seems reasonable to examine whether negative information is differentially successful in changing these two kinds of beliefs and in changing attitudes based differentially on such beliefs (Ohanian, 1990). One may argue that beliefs about competence and expertise are based relatively more on objectively verifiable data (Alwitt, Deighton, \& Grimm, 1991) obtained in a piecemeal fashion, which require some cognitive integration and elaboration before they can be used in a composite evaluation. If so, such beliefs should be more difficult to change via counterattitudinal messages that do not rely on reasoned argumentation, such as the negative political advertisements studied here. In contrast, because beliefs about character traits such as trustworthiness and likability are more felt and subjective, rather than derived from such cognitive integration of externally verifiable information (Geiger \& Reeves, 1991), they may well be more subject to change when presented with the kinds of derogatory personal references contained in negative personal advertising. A similar conceptual case was made by Edwards (1990, p. 211) regarding the ease with which cognitively based versus affectively based attitudes can be changed by counterattitudinal communications containing cognitive versus affective appeals. (We return to the issue of whether our character/competence distinction is equivalent to Edwards's cognitive/affective distinction later.) Finally, in their review of negativity and extremity biases in impression formation, using a category diagnosticity approach, Skowronski and Carlston (1989) argued that the negativity bias should be much stronger for morality judgments than for ability judgments. 
Consistent with our previous proposition, it has in fact been found in another advertising context that negative information does have an unequal effect on the competence and character dimensions of the overall evaluation of a source (Klebba \& Unger, 1983). In a field research study of Lee Iacocca, chief executive officer of Chrysler Corporation, Klebba and Unger found that perceptions of a source's character (e.g., Iacocca's likability) were more influenced by negative information (i.e., knowledge of Iaccoca's association with the Ford Pinto) than were perceptions of the source's competence (e.g., his expertise). The negative information appeared to have a neutralizing effect on the character aspects of his evaluation but not on the competence aspects of his evaluation. This finding suggests that negative political communications may very well have differential effects on the character and competence dimensions of beliefs about the target (the attacked candidate). Specifically, the target candidate's belief ratings on likability and trustworthiness may be more vulnerable to negative attacks than his or her expertise ratings. We thus include these distinct belief dimensions in our contingency model and propose Hypothesis 2 (H2):

H2: Beliefs about the candidate's character (e.g., likability and trustworthiness) will be more influenced by negative information than will beliefs about competence (e.g., expertise).

\section{Character Versus Competence Focus in Forming Overall Attitudes}

A contingency model of the differential effects of negative communication may also incorporate the related notion that overall attitudes toward political candidates could be formed on the basis of information that varies in its character/competence orientation, depending on the type (focus) of appraisal being made. Rothschild (1984) showed that when voters develop attitudes toward political candidates they use different criteria and information in such evaluation tasks depending, in part, on the voter's level of involvement in that political race. More recently, Krosnick (1988) showed that attitudes toward presidential candidates are based on the candidate's stand on policy issues only when the same policy attitudes are important and accessible - which does not always happen because voters, being cognitive misers and not always very interested in political issues, have accessible policy attitudes on only a handful of political topics. Voting studies also frequently conclude that "the electorate does not engage in careful policy analysis and comparison of the party platforms offered by the major parties of candidates" (Marcus, 1988, p. 752). In other words, there are many situations when voters form their attitudes toward political candidates on cues such as likability, not on performance-related and policy beliefs (Johnson-Cartee \& Copeland, 1991, p. 53). 
Given these situations, such a character or competence attitude-formation focus on the part of the evaluating voter ought to interact with the nature of the message (positive vs. negative) in the political communication. If negative political communications are more effective in modifying character beliefs (as argued in the previous section), and if such character beliefs shape overall attitudes more under some conditions (e.g., when the level of voter knowledge about or involvement with the race are low), then negative political communications ought to be more powerful in changing such character-driven overall attitudes. That is, although negative information in general may impact attitudes more strongly than positive information (in the opposite direction), this disproportionate impact of negative information may be even more pronounced when overall attitudes are character driven than competence driven.

There appears to be no direct support for this proposition in the vast literature on attitude dimensions or components (see McGuire, 1985, for a review). However, two research "streams" offer indirect support. First, the elaboration likelihood model (ELM) suggests that attitudes based more on less elaborated peripheral cues (concerning source credibility and likability) may be more subject to counterattack (easy to change) than attitudes based more on more elaborated, central performance-relevant information (Petty \& Cacioppo, 1986, pp. 192-193, citing unpublished research by Petty, Cacioppo, Haugtvedt, \& Heesaker). Although the ELM does not distinguish between positive and negative information in such counterattacks, one may argue that characterbased attitudes are analogous to attitudes formed via the ELM's peripheral route and should, therefore, be more vulnerable to counterattack in either case.

Second, to the extent that our character/competence distinction in attitude formation resembles Edwards's (1990) distinction between affective/cognitive bases for attitude formation, her finding that affectively based attitudes are more susceptible to change from counterattitudinal messages with affective appeals also tends to support our proposition if we consider both characterbased attitudes and negative advertising to be high in affective content. Using this interpretation, an (affective) negative advertisement is able to "make contact" with "matching information" if the attacked attitudes are (affectively) character-based, and it is thus successful in changing these attitudes, whereas such an (affective) advertisement fails to find matching information in (more cognitive) competence-based attitudes, and thus it is less persuasive. Based on the preceding rationale, we expect that:

H3: The attitude-changing power of negative advertising will be greater when overall attitudes are formed on the basis of character beliefs rather than competence beliefs.

Finally, if the character beliefs that lead to such character-based attitudes-such as warmth/likability and trustworthiness-continue to be more salient 
when the attacking advertisements are subsequently processed, the effect of negative communications on these character beliefs also ought to be strongest under such character attitude-formation conditions. Yi (1990a, 1990b) demonstrated that once a concept is primed or activated, its relative salience is enhanced, and the likelihood of its use in encoding subsequent information increases. Thus, we expect the character beliefs of warmth/likability and trustworthiness to be most salient, and hence most vulnerable to change from negative attacks, in character attitude-formation conditions. In conclusion:

H4: The power of negative advertising in changing the character beliefs of warmth/likability and trustworthiness will be strongest when overall attitudes are based relatively more on such character criteria.

\section{METHOD}

\section{Overview}

In this study, a political radio ad was utilized as the information source in the experimental design; it contained either positive (candidate-supportive) or negative (candidate-attacking) information, and it was aimed at respondents who had already formed overall attitudes toward the targeted candidate either on the basis of character (i.e., likability/trustworthiness) or on the basis of competence (i.e., perceived expertise). Although this focus on attitude formation can be expected to vary naturally across respondents in actual political races (partly as a function of naturally varying levels of the voter's motivational involvement with the race), we experimentally manipulated it in this study and used the setting of a local mayoral election. This race was selected because we believed it was one in which voters would find it natural to use either character or competence beliefs to form attitudes; thus, it allowed us to manipulate the nature of beliefs used to form overall attitudes. In addition to overall attitudes, the candidates were also rated on both character (likability/ trustworthiness) and competence (expertise) belief dimensions. ${ }^{1}$

\footnotetext{
${ }^{1}$ The study reported here also attempted to manipulate the issue versus image characteristics of the information in the attacking advertisement. The hypothesis investigated was that issueoriented information would behave the same way as information on competence traits, because both may be more cognitive in nature, whereas image-oriented information would parallel the results for character-oriented information if both were affectively driven. However, although pretests indicated that the desired issue versus image effect was achieved, manipulation checks in the main study suggested otherwise. As a consequence, this manipulation did not have any significant effects on the dependent variables of interest and is therefore not discussed. The effects for the other manipulations are thus pooled over this issue/image manipulation.
} 
We used two between-subjects factors in this experiment: Focus of thought in the formation of prior attitudes (two levels, character vs. competence) and valence of information in the test ad (two levels, positive/supportive vs. negative/attacking). In addition to the four between-subjects conditions, two control groups were also included; these control subjects received the thought focus manipulation but not the ad valence manipulation that was embedded in the political radio ads because they did not receive the test ad.

\section{Manipulations}

Prior overall attitudes. To test some of the hypotheses, respondents must have a prior attitude for one of the candidates. This was accomplished by having subjects read a newspaper editorial that described each candidate (written to reflect views of the editorial board) prior to their listening to a radio program segment that contained the test ad manipulation. In this newspaper editorial, one candidate was made to appear more favorable in terms of desired characteristics (e.g., experience, likability, and family life) than the other, though even the less favorably described candidate was described as a reasonable alternative. The editorial ended with an endorsement for the more favorably described candidate. To avoid confounding the test ad stimulus manipulation with this newspaper endorsement, the actual information in the editorial differed from that of any of the political commercials. For example, the editorial discussed the candidates' professional experience, but this topic was not incorporated in any of the radio ads.

To pretest this prior attitude manipulation, 28 respondents from the same subject population used in the main study read the newspaper editorial manipulations. To test the effectiveness of the editorial in generating preference for one of the candidates (Ken Anderson), paired $t$ tests were performed comparing the trait and attitude measures (alphas ranging from .86 to .97 ; these scales are described later) between Ken Anderson and the opposing candidate, Bert Lindsay. As desired, Ken Anderson was rated more favorably on each of the trait scales and on the overall attitude scale (all $p<.03$ ): Expertise, $t(22)=3.11, M=6.10$ versus 4.79; Warmth, $t(25)=2.43, M=$ 5.88 versus 5.11 ; Ethics, $t(25)=2.27, M=5.89$ versus 5.01 ; Credibility, $t(25)$ $=2.45, M=6.18$ versus 5.26; and Overall Attitudes, $t(27)=2.66, M=6.08$ versus 4.76. Note that the less favored candidate still had overall attitudes directionally greater than the midpoint of the 8-point scale $(4.76$, which is higher than 4.5). (The degrees of freedom in these tests and some subsequent analyses differ as a result of missing data.)

Competence versus character attitude-formation manipulation. The relative importance of competence- and character-driven thought in the forma- 
tion of the prior attitudes just described was manipulated in two fully confounded ways: via the instructions given to the subjects and within the newspaper editorial. Before reading the editorial, subjects were instructed to pay close attention to either the information about the candidates' skills and competence (competence focus) or their warmth and friendliness (character focus). In addition, the first paragraph of the editorial described either a troubled city environment and the need to elect a "mayor who has the skills and competence to tackle the immense problems" (competence focus) or a healthy city environment in need of a "mayor who has the warmth and friendliness to be respected and liked by all" (character focus).

The success of this attitude focus manipulation was pretested through a recall task given to subjects $(n=10)$ after the reading of the editorial. Subjects in the character focus treatment recalled more information about the candidates' personal character (calculated as number of character minus number of qualification items correctly recalled) than those in the competence focus treatment, $t(8)=3.5, M \mathrm{~s}=3.0$ for the character focus group and -0.6 for the competence focus group.

Test ad valence manipulations. Two types of political radio commercials, varying in valence (i.e., positive vs. negative information), were utilized to test the proposed hypotheses. ${ }^{2}$ Thus, each subject heard either a positive (candidate-supportive) or negative (candidate-attacking) political advertisement. The implied sponsor of the positive ad was the candidate favored in the newspaper editorial, Ken Anderson. The implied sponsor of the negative ad was the opponent, Bert Lindsay. In order to use equivalent positive and negative information in these advertisements, the positive statements were merely negated with as few word changes as possible. For example, "He supported the job development program . . ." became "He opposed the job development program ..." The ads were also purposefully designed to focus on issue- or image-related information that was not mentioned in the newspaper editorial's discussion (of the city's environment and the candidates' jobrelated experiences and family backgrounds).

The effectiveness of the valence manipulation was pretested. Forty-seven subjects heard two different sets of test commercials being considered for use. These ads were rated on individual indicators of valence (provided negative/ positive information, presented reasons for voting/for not voting for the can-

\footnotetext{
${ }^{2}$ Each of these advertisements (positive and negative) had two versions, utilizing either issue-oriented or image-oriented information. Although the issue ads dealt with topics such as job development, child care, and crime, the image ads dealt with topics of the candidates' tax returns, community involvement, and the number of years lived in the city. Because this issue/ image manipulation had no significant effect, the data were pooled over this manipulation, and it is not discussed further (see footnote 1).
} 
didate, and was/was not a mud-slinging ad). Multiple-item scales combining the appropriate indicators each had coefficient alphas exceeding .90 . Based on these scalar ratings, one set of two commercials was selected for final refinement and use. For this finally used set, analyses of variance (ANOVAs) showed that a main effect for valence emerged for the valence-dependent measure, $F(1$, $43)=461.73, p<.0001$. The positive commercial was perceived as being more positive $(M=8.14)$ than the negative commercial $(M=1.75)$. Other tests indicated that the commercials were perceived as equally strong (i.e., persuasive, convincing, important, of interest, understood, and easy to follow) and produced similar levels of recall (none of these effects were significant; $p>$ .05 ).

The commercials were equivalent in length (time) and amount of information (number of words). All commercials were professionally recorded by the same male radio announcer, unknown in the local area. This guaranteed equivalence across the spots, high-quality sound reproduction, and enhanced realism.

\section{Subjects}

A total of 187 eligible Texan voters, assigned randomly to the different conditions (i.e., 149 were exposed to one of the political test ads, and 38 were in the two control groups) participated in the main experiment. As in the pilot studies, administration of the experiment was performed via a shopping mall intercept method in the southwestern United States. Each participant was given a monetary incentive of $\$ 5.00$ as compensation for his or her time and effort. The median age of the sample was $30,51 \%$ were female, $60 \%$ had household incomes between $\$ 15,000$ and $\$ 50,000$ (median $=\$ 30,000$ ), and the median number of persons residing in the household was two.

\section{Procedure}

Each respondent performed the various experimental tasks in isolation. First, participants were instructed to read the instruction sheet (including a statement of the cover story for the experiment, "A Study of Political Voter Preferences"). Then, they read the newspaper editorial about the two candidates. Next, they listened to a radio program (10 min in length) in which the political communication(s) and filler commercials were embedded. When the program ended, the subjects were instructed to complete the questionnaire containing the dependent measures.

While listening to the radio program, each participant twice heard one of the two political communications resulting from the experimental manipulations. The total number of commercials embedded in the radio programs was the same across treatments, but subjects were either exposed to one political 
ad and two product (filler) ads, or three product ads (in the control conditions). In all instances, the 10-min radio segment began with one commercial break (containing two ads), followed by a segment from an unfamiliar talk show, and ended with a second commercial break (containing two ads). The experimental ad appeared once at the beginning of the tape and again at the end of the tape, both to stimulate more reliable cognitive responses and to eliminate primacy and recency confounds.

All experimental treatments were administered randomly, and recruitments were conducted over a 2-week period during all mall operating hours. The administrators were blind to the individual treatment assignments and research hypotheses.

Measures. Prior to hearing the radio ads, subjects recalled the newspaper editorial content (unaided), and then they assessed the extent to which they thought it focused on character (warmth, friendliness, and likability; $\alpha=.93$ ) versus competence (competence, skills, and expertise, $\alpha=.92$ ) information. These latter scales served both as manipulation checks and as a means of reinforcing the character/competence attitude-formation manipulation.

Immediately after listening to the program segment, cognitive responses were collected through a verbal protocol using standard instructions (cf. Batra \& Ray, 1986). These thoughts and feelings were coded independently by at least two judges as either ad cognitions (positive, negative, or neutral), imagerelated candidate thoughts (positive or negative), issue-related candidate thoughts (positive or negative), other candidate thoughts (positive or negative), neutral candidate thoughts, feelings (positive, negative, or neutral), or other thoughts. Coder agreement was $95 \%$, and all disagreements were resolved by discussion.

Measures of the underlying trait dimensions were primarily derived from those identified by Ohanian (1990) and Abelson, Kinder, Peters, and Fiske (1982). Perceptions of each candidate were assessed via 11 indicators designed to capture the dimensions of competence/skills, likability/warmth, and trustworthiness/ethics. The 11 individual indicators used to measure perceptions for each candidate were first analyzed via exploratory principal components analysis with varimax rotation. When the scree test was used, three distinct factors emerged (with the third having an eigenvalue less than 1.0), and their factor loadings were consistent with the three constructs presented earlier. An Expertise factor loaded strongest on items of qualification, ability, skills, and expertise; a Likability factor loaded strongest on items of likable, warm, nice, and friendly; and a Trustworthiness factor loaded strongest on items of trustworthy, honest, and ethical. If only the two factors with eigenvalues greater than 1.0 were retained and rotated, the items for trustworthiness and likability loaded together on one factor, whereas the items for Expertise comprised the second factor. The latter results support our conceptual treatment to this 
point, in which we have distinguished between competence (expertise) on the one hand and character (comprising both likability and trustworthiness) on the other.

However, for the sake of consistency with the three-dimensional dimensional structure reported by Ohanian (1990) and to allow for the emergence of possibly separate results for trustworthiness and likability, we decided to proceed with the development of three separate scales for Trustworthiness/Ethics, Warmth/Likability, and Competence/Skills. Confirmatory factor analyses (CFA) were used to verify the convergent and discriminant validity of the three proposed scales. (Details of these tests are omitted for brevity and are available from Pamela M. Homer.)

Following this CFA-based test of scale validity, summation of the appropriate items within each scale produced reliable scales for competence/skills (incompetent/competent, unqualified/qualified, low ability/high ability, and not expert/expert), warmth/likability (not likable/likable, cold/warm, not nice/nice, and unfriendly/friendly), and trustworthiness/ethics (untrustworthy/trustworthy, dishonest/honest, and unethical/ethical) for the target candidate (alphas ranging from .88 to .94 ). These construct development procedures were replicated for the opponent-related measures and produced equivalent results.

Other items in the questionnaire included 8-point scalar measures of overall attitudes toward the target anchored as follows: negative (1) to positive (8), dislike (1) to like (8), and unfavorable (1) to favorable (8). Summing these items yielded highly reliable scales $(\alpha=.95)$. Finally, other measures gauged the intent to vote in the election and the intent to vote for each candidate (unlikely/likely), unaided recall of ad content, manipulation checks for ad valence (described in the Manipulations section) familiarity with each candidate (not at all familiar/very familiar), task involvement (not at all involved/very involved), demographic information, and bogus questions designed to disguise the intent of the experiment. These bogus questions referred to the filler commercials and program content (i.e., they were consistent with the cover story). At the conclusion, subjects were asked to list their thoughts and feelings during the experiment and to complete items designed to determine whether they knew the purpose of the experiment. It was apparent that no participants were aware of the purpose or guiding hypotheses.

\section{RESULTS}

\section{Manipulation Checks}

Character versus competence basis of prior attitudes. Consistent with the pilot studies, respondents in the character attitude-formation treatment recalled relatively more information about the candidate's personal character 
than about his qualifications ( $M$ number of character items - number of qualification items $=2.08$; this is significantly different from 0$), t(185)=$ $10.89, p<.01$. This difference measure mean was significantly lower in the competence-focus treatment, $t(185)=9.25, p<.001$, where the mean was -0.20 , which was not significantly different from $0, t(185)=1.29$, ns. As desired, the proportion of information that was character related was higher for those in the character-focus group than in the competence-focus group ( $M$ $=.62$ vs. .47$), t(185)=7.49, p<.001$, whereas the proportion of qualification-related information recalled was greatest in the competence-focus group $(M=.37$ vs. .53$), t(185)=7.67, p<.001$. Additional support for the effectiveness of the attitude-formation focus manipulation was indicated by analyses of scale items designed to measure the extent to which the subjects self-reported their focusing on character versus competence information. Those in the character focus treatment reported attending relatively more to the information about warmth and friendliness rather than the information about competence and skills (calculated as warmth/friendliness - competence/skills information; $M=-0.35)$ than those in the competence focus treatment $(M=-1.19), t(186)=3.32, p<.001$.

Valence of information in ad. An ANOVA revealed that the negative appeal ads $(M=7.01)$ were distinctly perceived as containing more negative information, reasons for not voting for the attacked candidate, and "mudslinging" $(\alpha=.95)$ than the positive appeal ads $(M=1.71), F(1,143)=$ 743.91, $p<.001$. As another check, indices were created to assess the net valence of candidate-related thoughts and ad-related thoughts that emerged from the thought-listing task (calculated as positive thoughts - negative thoughts). As expected, the negative valence ads generated fewer supportarguments minus counterarguments about the attacked candidate $(M=$ $-0.72)$ than the positive communications $(M=1.39), F(1,147)=53.52$, $p<.001$, when image, issue, and other candidate thoughts were summed. The ad-related thought index behaved similarly, $F(1,147)=33.39, p<.001$ $(M \mathrm{~s}=0.00$ and -1.52 for the positive and negative appeal ads, respectively). The individual thought measures paralleled these aggregate findings: For each type of candidate-related thought (image, issue, and other), more positive thoughts were expressed for the positive appeals and more negative thoughts for the negative appeals; positive ad thoughts were higher for the positive appeals; and negative ad thoughts tended to occur in the negative ad treatments.

Also note that, as gauged by our covariate scalar measures, subjects were not previously familiar with either our target or opponent candidates $(M \mathrm{~s}=$ 1.30 and 1.26 , respectively). This is not surprising because our stimuli dealt with fictitious candidates, but it does make clear that any effects were due to the experimental manipulations, not preexisting beliefs. 
Tests of Hypotheses: Overall Multivariate Analysis of Variance (MANOVA)

Because our primary interest is in the change in attitudinal judgments in the test groups due to ad exposure (both positive and negative effects), difference variables for each scale (overall attitudes toward the candidate, warmth/ likability, trustworthiness/ethics, and competence/skills) were created by subtracting each subject's individual test group score from the mean score of the no ad exposure (control) groups. ${ }^{3}$ Such difference or gain scores allow for the computation not only of relative treatment effects (of positive vs. negative information) but also of their absolute magnitude (positive information vs. no information), assuming that the sample mean of the control group is the control group mean. (For a discussion, see Cook \& Campbell, 1979, chapter 4.) These difference/gain score variables serve as surrogates to the more conventional approach in which experimental treatment and control groups are compared via planned contrasts. Alternative analyses, using these planned contrasts instead of using just the difference scores, were also performed; they yielded the same main and interaction effects, but they are not reported here.

The four difference variables were then incorporated in a MANOVA with the two independent variables (i.e., focus of attitude-forming thought and valence of ad information). This two-factor MANOVA identified a Focus $x$ Valence interaction (Wilks's lambda $=.923$ ), $F(4,139)=2.90, p=.024$, and a valence main effect (Wilks's lambda $=.711), F(4,139)=14.12, p<.001 .{ }^{4}$ Univariate ANOVAs and planned comparisons (Duncan's Multiple Range Test) among individual cell means were performed next to assess the guiding hypotheses. These findings are summarized in Tables 1 and 2.

\section{$\mathrm{H} 1$ and $\mathrm{H} 2$}

Evidence of the power of negative information over similar positive information is overwhelming. The presence of a valence effect emerged for all the dependent variables of concern (as measured by differences from the appropriate control group).

Supporting H1, a valence effect emerged for the difference in overall attitudes, $F(1,142)=15.67, p<.001(M \mathrm{~s}=-0.02$ and -1.19 for the positive and negative appeal, respectively). Whereas the positive appeal did not raise

\footnotetext{
${ }^{3}$ Because the difference measures were created by subtracting the test group data from the appropriate control group, these analyses are based on data from those subjects exposed to one of the treatment ads (minus those observations omitted due to missing data).

${ }^{4}$ Following Marcus (1988), a second MANOVA was also performed using a comparative perceptions approach, in which each of the target candidate's (i.e., Ken Anderson's) judgment scale scores were subtracted from those of the appropriate opponent (i.e., Bert Lindsay). These results are omitted for brevity, but they parallel those from the standard approach.
} 
TABLE 1

Effects of Focus of Thought and Ad Valence on Beliefs and Overall Attitudes

\begin{tabular}{lrc}
\hline Dependent Variable & $F$ & $p$ \\
\hline Overall attitudes & & \\
Focus & 0.78 & .378 \\
Valence & 15.67 & .000 \\
Valence $\times$ Focus & 5.49 & .020 \\
Competence/skills beliefs & & \\
Focus & 0.51 & .478 \\
Valence & 4.94 & .028 \\
Valence $\times$ Focus & 1.97 & .162 \\
Warmth/likability beliefs & & .474 \\
Focus & 0.51 & .000 \\
Valence & 38.84 & .008 \\
Valence $\times$ Focus & 7.13 & .056 \\
Trustworthiness/ethics beliefs & & .000 \\
Focus & 3.71 & .001 \\
Valence & 41.99 & \\
Valence $\times$ Focus & 10.90 & \\
\hline
\end{tabular}

TABLE 2

Treatment Means for the Valence $\times$ Focus of Thought Interaction Effect on the Dependent Measures

\begin{tabular}{lcr}
\hline Dependent Variable & Negative & Positive \\
\hline Overall attitudes & & \\
$\quad$ Character focus & -1.63 & 0.19 \\
$\quad$ Competence focus & -0.70 & -0.23 \\
Competence/skills beliefs & & \\
$\quad$ Character focus & -0.85 & 0.02 \\
$\quad$ Competence focus & -0.34 & -0.15 \\
Warmth/likability beliefs & & \\
$\quad$ Character focus & -1.72 & 0.41 \\
$\quad$ Competence focus & -0.91 & -0.06 \\
Trustworthiness/ethics beliefs & & \\
$\quad$ Character focus & -1.84 & 0.58 \\
Competence focus & -0.55 & 0.24 \\
\hline
\end{tabular}

attitudes compared to the control group, the negative appeal clearly succeeded in lowering attitudes.

Although this finding could be viewed as merely replicating Lau's (1985) work, it has the important advantage of being experimental, not merely correlational. However, as an experimental result, it could be challenged by a potential confound. Because our manipulation created mildly positive (rather than exactly neutral) attitude ratings for the targeted candidate prior to exposure to the two test ads, it could be argued that the positive ad had no room 
to increase the candidate's rating (i.e., a ceiling effect existed), whereas the negative ad had considerable room to reduce it. Although such a rival hypothesis cannot be totally ruled out, the data argue against it in two ways. First, the preexposure (control group) mean overall attitude was 5.91 on the 8-point scale, clearly allowing the overall attitudes of the positive appeal subjects to get more positive, but this did not happen. Second, if a ceiling effect existed for the positive appeal, such a ceiling effect should have occurred for all dependent variables and all manipulation cells. However, although the positive appeal did not succeed in raising overall attitudes over the control, when aggregated over the character and competence attitude-focus groups, it did succeed in raising the trustworthiness and likability belief scores over the control (up by 0.58 and 0.41 , respectively, both significantly greater than 0 at $p<.05$ ) for the character attitude group alone. Thus, a ceiling effect rival hypothesis appears implausible.

Regarding H2, analyses showed first that perceptions of the target candidate on all three dimensions of competence/skills, warmth/likability, and trustworthiness/ethics were in each case negatively affected by the negative ad $(M \mathrm{~s}=-0.62,-1.34$, and -1.19 , respectively), but they remained stable $(M \mathrm{~s}$ $=-0.07,0.18$, and 0.40 , respectively) - and statistically equal to the no exposure controls-after exposure to the positive message $F_{\mathrm{s}}(1,142)=4.94$, $p=.028 ; 38.84, p<.001$; and $41.99, p<.001$, respectively.

To determine if perceptions of the two character belief characteristics are more influenced by negative information than perceptions of the competence belief characteristics (H2), initial assessments of the relative contribution of the valence effect for each belief characteristic compared the $F$ values and eta coefficients across the three ANOVAs. As hypothesized, ad valence contributed relatively more to the explained variance for perceptions of warmth/ likability, $F(1,142)=38.34, p<.001$, eta $=.46$, and trustworthiness/ethics, $F(1,142)=41.99, p<.001$, eta $=.46$, than it did for perceptions of the candidate's competence/skills, $F(1,142)=4.94, p=.028$, eta $=.19$. A repeated measures MANOVA was also performed to test $\mathrm{H} 2$. The two character belief scales were first averaged. This summated measure and the Competence Belief scale were then treated as a within-subjects Belief measure (i.e., these two belief measures were input as dependent variables). Supporting H2, a within-subjects Belief $\times$ Ad Valence interaction emerged, $F(1,143)=26.58$, $p=.000$, indicating that character beliefs $(M \mathrm{~s}=0.30$ and -1.25 for the positive and negative appeal ads, respectively) are more influenced by negative communications than competence beliefs $(M \mathrm{~s}=-0.07$ and -0.59 , respectively). ${ }^{5}$

Regression analyses were also performed to investigate the relative influence

\footnotetext{
${ }^{5}$ Nondifferenced test and control group data produced equivalent findings.
} 
that positive and negative thoughts (measured from the verbal protocols) had on the dependent variables in $\mathrm{H} 1$ and $\mathrm{H} 2$. Tests showed that subjects in the positive information treatments understandably did not have adequate variation in the number of negative thoughts reported $(83 \%$ of these subjects had 0 negative thoughts; $M$ number of negative thoughts $=.21$ ). These regression analyses were therefore limited to subjects in the negative thought condition, who did report a much larger number of negative thoughts $(M=.91$, with only $53 \%$ of these subjects reporting 0 negative thoughts). For this negative thought sample, positive and negative candidate-related thoughts were designated as the independent variables in regression models that used the overall attitude and three belief measures as dependent variables to test the hypothesis that negative information would influence them more than positive information. The relative contribution of the negative and positive thoughts was tested by comparing the full model to the reduced model containing only the negative thoughts.

According to the incremental $F$ tests (Hays, 1988), the full model explains essentially the same amount of the variance in overall attitudes, warmth/likability, and trustworthiness/ethical candidate perceptions as the model that includes only negative thoughts $\left(F_{\mathrm{ADD}}=1.95,1.73\right.$, and 2.57 , all $p s>.05$, for overall attitudes, warmth/likability beliefs, and trustworthiness/ethics beliefs, respectively). In contrast, the incremental $F$ test for competence/skills beliefs indicated that both positive and negative thoughts made a contribution here $\left(F_{\mathrm{ADD}}=5.39\right)$. These results thus indicate that, although negative thoughts influence (help to determine) all of the dependent variables, positive thoughts make a predictive contribution only to competence/skills beliefs (see Table 3). (These conclusions stay the same if the full models are compared with models containing only positive thoughts, also shown in the table.) Thus, $\mathrm{H} 2$, which deals with the differential effectiveness of negative information, is supported here in an unexpected way. We had expected that negative information would have stronger effects on character than on competence beliefs; we found that this was true in a relative, rather than an absolute sense, in that, although positive thoughts impacted (raised) only the competence beliefs, negative information impacted (decreased) all.

\section{$\mathrm{H} 3$ and $\mathrm{H} 4$}

H3 (i.e., that the dominance of negative information would be greater when attitudes were formed on the basis of character rather than competence beliefs) was examined using an ANOVA. A Focus of Attitude-Forming Thought $x$ Ad Information Valence interaction emerged for overall attitude toward the targeted candidate, $F(1,142)=5.49, p=.020$. Although negative advertising reduced overall attitudes by 0.70 under a competence attitude-forming thought 
TABLE 3

Impact of Candidate-Related Thoughts on Character Perceptions and Overall Attitudes

\begin{tabular}{lccc}
\hline & \multicolumn{3}{c}{ Standardized Coefficients } \\
\cline { 2 - 4 } Dependent Variable & $\begin{array}{c}\text { Positive } \\
\text { Thoughts }\end{array}$ & Negative \\
\hline Overall attitudes & .19 & $-.47^{*}$ & $R^{2}$ \\
& -17 & $-.47^{*}$ & .27 \\
Competence/skills & $.25^{* *}$ & $-.39^{*}$ & .25 \\
& $-24^{* *}$ & $-.38^{*}$ & .03 \\
Warmth/likability & .14 & $-.41^{*}$ & .21 \\
& -13 & $-.41^{*}$ & .15 \\
Trustworthiness/ethics & .19 & $-.37^{*}$ & .07 \\
& -17 & $-.37^{*}$ & .18 \\
& -16 & - & .02 \\
& & & .17 \\
& & & .14 \\
& & & .03 \\
\hline
\end{tabular}

${ }^{*} p<.001$.

${ }^{* *} p<.05$.

focus, it reduced it by more than twice as much (1.63) when the prior attitudes were based on character beliefs (see Table 2).

In testing $\mathrm{H} 4$, interactions similar to the last one also emerged for perceptions of the target candidates' warmth/likability, $F(1,142)=7.13, p<.01$, and trustworthiness/ethics, $F(1,142)=10.90, p<.001$. In each of these interactions, scores for the two character beliefs were lower for the negative appeal ad than the positive appeal ad if the attitude-formation focus was character, but this was not true when the focus was on competence (see Table 2 for a summary of the treatment means). No such interaction occurred for the Competence/Skills Belief scales. These results therefore support $\mathbf{H 4}$.

\section{DISCUSSION}

We attempted to develop a contingency model for the counterattitudinal effectiveness of negative political communications by examining whether negative political communications were more effective in some contexts than in others. Our results, in the context of political advertising, indicate that, although negative political messages indeed appear to have more attitude-changing power overall than positively valenced advertising, this main effect needs to be qualified by an important interaction. Specifically, the Thought Focus $x$ Valence interaction indicates that this disproportionate weight of negative information is greatest when the voter's attitude-forming thoughts are focused 
more on the attacked candidate's character (likability and trustworthiness) than his or her competence (expertise). Consistent with this interaction, the negative attacking ad also appeared to have a greater effect on these character beliefs (likability and trustworthiness) than on the expertise belief, and this effect of the negative ad was greatest when overall attitudes were formed on a character-focused basis. Both of these effects corroborate Skowronski and Carlston's (1989) category diagnosticity approach that postulates that attributes perceived as more diagnostic influence impression formation more than less diagnostic attributes and that negative cues are perceived as more diagnostic for morality/character cues than they are for ability/competence judgments.

These results are important from both a public policy/political advertising viewpoint and from that of theory development about attitude structure. From the political advertising/public policy standpoint, our main effect result is important because it provides experimental support for the greater effectiveness of negative information that thus far has been identified only through correlational means (e.g., Lau, 1982, 1985). Thus, because it offers greater evidence of a cause-and-effect relation, our main effect result is more than a mere replication. Note, however, that the disproportionate effect of negative information observed here (as in other studies) is a relative effect, taking place within the context of the positive appraisal of both the candidates created by our attitude-forming manipulation. The effect may have been of a different magnitude, and possibly even in a different direction, if we had initially set up a negative initial appraisal of both candidates. Further, our methodological choices (e.g., the use of a mayoral election with both candidates being equally well-known) may limit the generalizability of our results to any particular "real" political race.

In addition, our interaction results are very important in that they show the greatest effect for negative political communications to be on characterfocused voters-those who place highest importance on character beliefs and use such beliefs more strongly in forming overall political attitudes. It appears that such negative political communications are less likely to be effective in changing attitudes toward the attacked candidate when such prior attitudes are formed on the basis of the candidate's perceived qualifications and expertise. This important moderating effect of a character/competence focus should help explain why negative political communications have appeared to work only in some political contexts and not in others (the literature on this was reviewed earlier). Although the character/competence variable studied here may not be the only one that explains this situational effectiveness of negative political communications, or even be the most important one, it offers some insight into why such communications have the mixed success they apparently do. For instance, one way of explaining why George Bush's negative advertising campaign was apparently very successful against Michael Dukakis in 1988, 
but not against Bill Clinton in 1992, is that recession-battered 1992 voters may have formed their attitudes based much more on a candidate's perceived competence than on character compared to the use of these criteria by voters in 1988.

Our results also have some implications for the study of attitude structure and change. They show once again that the susceptibility of attitudes to change do appear to depend significantly on the nature of the information used to form that attitude, which interacts with the nature of the information present in the counterattitudinal communication. We found that character-focused individuals were more capable of assimilating or accommodating the new negative information than their competence-focused counterparts. (It was our character-focused subjects who showed most change with the negative ads.) This result is somewhat similar to that of Petty, Cacioppo, Haugtvedt, and Heesaker (cited in Petty \& Cacioppo, 1986, pp. 192-193), who showed that attitudes based on peripheral cues were less resistant to change (from any kind of counterattitudinal communication) than attitudes based on a considered evaluation of domain-relevant message arguments. Our result is even more similar to that of Edwards (1990), who showed that attitudes formed affectively were more likely to change when confronted with a counterattitudinal message using affective (rather than cognitive) information, whereas attitudes formed cognitively were likely to change about equally when confronted with either affective or cognitive messages. (Note, however, that our results and Edwards's are not consistent with those of Millar \& Millar, 1990. Although the reasons for these differences are unclear, they may be due to methodological differences such as the more public commitment of attitudes by subjects in Millar and Millar's study.)

Thus, our result supports the conceptual arguments made by Zanna and Rempel (1988), who argued that attitudes are based differentially on different kinds of information (beliefs, feelings, and/or past behavior) and that attitudes based differentially on these sources of information are also differentially resistant to persuasive communications (p. 327). As further developed by Edwards (1990), the key idea here is that influence attempts must match an attitude's origin in order to make contact with it: If the initial (target) attitudes are based primarily on affect, then it is likely (and Edwards's results show) that these attitudes are most likely to change if the counterattitudinal communication is also affect based. (A similar argument is also made by Zajonc \& Markus, 1982, p. 127.) If character-based attitudes and negative political advertising both share a focus on the same type of trait information, our result is analogous and thus allows such a match. We acknowledge here that the matching idea just discussed is subject to various limitations and boundary conditions: For instance, the attitudes must not subsequently acquire a dominant cognitive character, and the initial affect must still be accessible, primary, and dominant; if the initial affect becomes disassociated from the final attitude, 
then cognitively based persuasion attempts may well be more successful at persuasion. The need for such a match may also vary with the strength with which the initial attitude is held (see the discussions in Edwards, 1990, and Millar \& Millar, 1990).

One may ask whether our own character versus competence distinction is really any different from the cognitive versus affective distinction used by Edwards (1990) and in much prior attitude research. After all, as we argued earlier regarding construct development, the likability and trustworthiness of the political candidate involved engender the feeling of a subjective bond between the voter and the candidate, an important characteristic of an affectively formed attitude (cf. Zajonc, 1980, p. 157), whereas an attitude formed on the basis of an appraisal of the candidate's competence and qualifications ought to involve a relatively more effortful integration of different pieces of objectively verifiable information, which suggests a more cognitive basis of effortful formation. If this position is accepted, then our results reinforce Edwards's (1990).

Clearly, however, such suggestions need to be investigated empirically in future research, using measures of experienced feeling. Nevertheless, our study, like many others, clearly supports the underlying idea that all attitudes are not alike, but instead they are complex multidimensional bundles of cognitive appraisals combined with precognitive affects. Studies of attitude formation and change need to be much more explicit regarding the specific attitudinal dimension(s) being investigated.

\section{ACKNOWLEDGMENT}

This project was funded by a Faculty Research Grant from the University of Texas at Austin to Pamela M. Homer.

\section{REFERENCES}

Abelson, R., Kinder, D., Peters, M., \& Fiske, S. (1982). Affective and semantic components in political person perception. Journal of Personality and Social Psychology, 42, 619-630.

Alwitt, L. F., Deighton, J., \& Grimm, J. (1991). Reactions to political advertising depend on the nature of the voter-candidate bond. In F. Biocca (Ed.), Television and political advertising: Vol. 1. Psychological processes (pp. 329-350). Hillsdale, NJ: Lawrence Erlbaum Associates, Inc.

Anderson, N. (1965). Averaging versus adding as a stimulus-combination rule in impression formation. Journal of Personality and Social Psychology, 2, 1-9.

Batra, R., \& Ray, M. L. (1986). Affective responses mediating acceptance of advertising. Journal of Consumer Research, 13, 234-249.

Birnbaum, M. (1972). Morality judgments: Tests of an averaging model. Journal of Experimental Psychology, 93, 35-42. 
Cook, T. D., \& Campbell, D. T. (1979). Quasi-experimentation: Design and analysis issues for field settings. Chicago: Rand McNally.

Edwards, K. (1990). The interplay of affect and cognition in attitude formation and change. Journal of Personality and Social Psychology, 59, 202-216.

Feldman, S. (1966). Motivational aspects of attitudinal elements and their place in cognitive interaction. In S. Feldman (Ed.), Cognitive consistency (pp. 75-87). New York: Academic.

Garramone, G. M. (1984), Voter responses to negative political ads. Journalism Quarterly, 61, $250-259$.

Garramone, G. M., Steele, M. E., \& Pinkleton, B. (1991). The role of cognitive schemata in determining candidate characteristic effects. In F. Biocca (Ed.), Television and political advertising: Vol. 1. Psychological processes (pp. 311-328). Hillsdale, NJ: Lawrence Erlbaum Associates, Inc.

Geiger, S. F., \& Reeves, B. (1991). The effects of visual structure and content emphasis on the evaluation and memory for political candidates. In F. Biocca (Ed.), Television and political advertising: Vol. 1. Psychological processes (pp. 125-143). Hillsdale, NJ: Lawrence Erlbaum Associates, Inc.

Hass, R. G. (1981). Effects of source characteristics on cognitive responses and persuasion. In R. Petty, T. Ostrom, \& T. Brock (Eds.), Cognitive responses in persuasion (pp. 141-172). Hillsdale, NJ: Lawrence Erlbaum Associates, Inc.

Hays, W. L. (1988). Statistics. New York: Holt, Rinehart \& Winston.

Hovland, C. I., Janis, I. K., \& Kelley, H. H. (1953). Communication and persuasion. New Haven, CT: Yale University Press.

James, K. E., \& Hensel, P. J. (1991). Negative advertising: The malicious strain of comparative advertising. Journal of Advertising, 20, 53-69.

Jamieson, K. (1984). Packaging the presidency. New York: Oxford University Press.

Johnson-Cartee, K. S., \& Copeland, G. A. (1991). Negative political advertising: Coming of age. Hillsdale, NJ: Lawrence Erlbaum Associates, Inc.

Kinder, D. R. (1986). Presidential character revisited. In R. R. Lau \& D. O. Sears (Eds.), Political cognition: The Nineteenth Annual Carnegie Symposium on Cognition (pp. 233-255). Hillsdale, NJ: Lawrence Erlbaum Associates, Inc.

Klebba, J., \& Unger, L. (1983). The impact of negative and positive information on source credibility in a field setting. In R. Bagozzi \& A. Tybout (Eds.), Advances in consumer research (Vol. 10, pp. 11-16). Ann Arbor, MI: Association for Consumer Research.

Krosnick, J. A. (1988). The role of attitude importance in social evaluations: A study of policy preferences, presidential candidate evaluations, and voting behavior. Journal of Personality and Social Psychology, 55, 196-210.

Lau, R. (1982). Negativity in political perception. Political Behavior, 4, 353-378.

Lau, R. (1985). Negativity effects in political behavior. The American Journal of Political Science, 29, 119-138.

Lodge, M., McGraw, K. M., \& Stroh, P. (1989). An impression-driven model of candidate evaluation. American Political Science Review, 83, 399-419.

Marcus, G. E. (1988). The structure of emotional response: 1984 presidential candidates. American Political Science Review, 82, 737-762.

McGuire, W. J. (1969). The nature of attitudes and attitude change. In G. Lindzey \& E. Aronson (Eds.), Handbook of social psychology (2nd ed., Vol. 3, pp. 136-314). Reading, MA: AddisonWesley.

McGuire, W. J. (1985). Attitudes and attitude change. In G. Lindzey \& E. Aronson (Eds.), Handbook of social psychology (3rd ed., Vol. 2, pp. 233-346). New York: Random House.

Merritt, S. (1984). Negative political advertising: Some empirical findings. Journal of Advertising. 13, 27-38.

Millar, M. G., \& Millar, K. U. (1990). Attitude change as a function of attitude type and argument type. Journal of Personality and Social Psychology, 59, 217-228. 
Miller, A. H., Wattenberg, M. P., \& Malanchuk, O. (1986). Schematic assessments of presidential candidates. American Political Science Review, 80, 521-540.

Mizerski, R. W. (1982). An attribution explanation of the disproportionate influence of unfavorable information. Journal of Consumer Research, 9, 301-310.

Ohanian, R. (1990). Construction and validation of a scale to measure celebrity endorsers' perceived expertise, trustworthiness, and attractiveness. Journal of Advertising, 19, 39-53.

Petty, R. E., \& Cacioppo, J. T. (1986). Communication and persuasion: Central and peripheral routes to attitude change. New York: Springer-Verlag.

Richey, M., Koenigs, R., Richey, H., \& Fortin, R. (1975). Negative salience in impressions of character: Effects of unequal proportions of positive and negative information. Journal of Social Psychology, 97, 233-241.

Rothschild, M. L. (1984). Perspectives on involvement: Current problems and future directions. In T. Kinnear (Ed.), Advances in consumer research (Vol. 11, pp. 216-217). Provo, UT: Association for Consumer Research.

Skowronski, J. J., \& Carlston, D. E. (1989). Negativity and extremity biases in impression formation: A review of explanations. Psychological Bulletin, 105, 131-142.

Surlin, S., \& Gordon, T. (1977). How values affect attitudes toward direct reference political advertising. Journalism Quarterly, 54, 89-98.

Taylor, P. (1986, October 5). Negative ads becoming powerful political force. The Washington Post, pp. Al, A6-A7.

Tinkham, S., \& Weaver-Lariscy, R. A. (1991). Advertising message strategy in US congressional campaigns: Its impact on election outcome. Current Issues and Research in Advertising, 13, 207-226.

Weinburger, M., Allen, C., \& Dillon, W. (1981). Negative information: Perspectives and research directions. In K. Monroe (Ed.), Advances in consumer research (Vol. 8, pp. 398-404). Ann Arbor, MI: Association of Consumer Research.

Yi, Y. (1990a). Cognitive and affective priming effects of the context for print advertisements. Journal of Advertising, 19, 40-48.

Yi, Y. (1990b). The effects of contextual priming in print advertisements. Journal of Consumer Research, 17, 215-222.

Zajonc, R. B. (1980). Feeling and thinking: Preferences need no inferences. American Psychologist, $35,151-175$.

Zajonc, R. B., \& Markus, H. (1982). Affective and cognitive factors in preferences. Journal of Consumer Research, 9, 123-131.

Zanna, M. P., \& Rempel, J. K. (1988). Attitudes: A new look at an old concept. In D. Bar-Tal \& A. Kruglanski (Eds.), The social psychology of knowledge (pp. 315-334). New York: Cambridge University Press.

Accepted by Gerald Gorn. 